FERREIRA, M.D.; FRANCO, A.T.O.; TAVARES, M. Técnicas de colheita para tomate de mesa. Horticultura Brasileira, Brasília, v.23, n.4, p.1018-1021, out-dez 2005.

\title{
Técnicas de colheita para tomate de mesa ${ }^{1}$
}

\section{Marcos David Ferreira²; André T.O. Franco ${ }^{3}$; Marcelo Tavares ${ }^{4}$}

${ }^{2 / U N I C A M P, ~ F a c . ~ E n g . ~ A g r i ́ c o l a, ~ C . ~ P o s t a l ~ 6011, ~ 13083-875 ~ C a m p i n a s-S P ; ~ E-m a i l: ~ m a r c o s . f e r r e i r a @ a g r . u n i c a m p . b r ; ~}{ }^{3 / U N I C A M P, ~ F a c . ~}$ Eng. Alimentos, C. Postal 6121, 13081-970 Campinas-SP; ${ }^{4}$ UFU, Fac. Matemática, Bairro Sta. Mônica, 38408-100 Uberlândia-MG

\section{RESUMO}

Comparou-se a qualidade de frutos provenientes de colheita utilizando cestas de bambu e sacolas de lona plástica em campos de produção na região de Mogi-Guaçu, SP. Utilizou-se como testemunha frutos não submetidos ao manuseio. O delineamento utilizado foi inteiramente casualizado em esquema fatorial (sistemas de colheita $\mathrm{x}$ dias após a colheita) com três repetições. Foram observados o tempo de colheita, incidência de danos físicos (\%) originados no campo e/ou no processo de colheita, perda de masa (\%) durante o armazenamento, e a qualidade visual após armazenamento por 21 dias a temperatura ambiente $\left(23^{\circ} \mathrm{C}\right)$. $\mathrm{O}$ tempo necessário para realizar a colheita no mesmo número de plantas utilizando-se cesta de bambu foi superior em $20 \%$, em relação à sacola plástica. A incidência de danos físicos (\%) e perda de massa (\%), apesar de maiores nos frutos colhidos com sacola, não foram significativamente diferentes dos colhidos com cestas de bambus. Observou-se maior perda de massa (\%) durante o armazenamento nos frutos colhidos utilizando-se sacolas de lonas plásticas. Após armazenamento por 21 dias, frutos colhidos com sacola plástica apresentavam maiores perdas do que aqueles colhidos utilizando-se cestas de bambu, principalmente devido a danos físicos e podridões.

Palavras-chave: Lycopersicon esculentum, cestas de bambu, sacolas plásticas, danos físicos, qualidade dos frutos.

\begin{abstract}
Harvesting methods for fresh market tomatoes

Quality of tomato fruits harvested using traditional bamboo baskets was compared to fruits harvested using harvest bags in the Mogi Guaçu region, São Paulo State, Brazil. Fruits not submitted to handling were used as control. The trial was totally randomized (harvest system $\mathrm{x}$ days after harvest) in a factorial design. The observed data were time spent for each harvest operation, mechanical injury (\%) caused either in the field or/and in the process of harvesting, weight loss (\%) during storage and final quality of fruits after storage for 21 days at room temperature $\left(23^{\circ} \mathrm{C}\right)$. The time necessary for the harvest with bamboo baskets was $20 \%$ higher than using plastic bags. The results showed that weight loss (\%) and injury incidence (\%) using harvesting bags were higher than the harvest using bamboo baskets. Weight loss (\%) was higher in fruits harvested with plastic bags during storage. After storage during 21 days, tomatoes harvested using plastic bag showed higher losses than fruits picked using bamboo baskets due to mainly mechanical injury and diseases.
\end{abstract}

Keywords: Lycopersicon esculentum, bamboo basket, harvesting bags, mechanical injury, fruit quality.

\section{(Recebido para publicação em 25 de junho de 2004 e aceito em 31 de julho de 2005)}

A produção anual brasileira de tomate representa $3 \%$ de toda a produção mundial, colocando o país em nono lugar em toneladas produzidas (FNP CONSULTORIA \& COMÉRCIO, 2001). Em 2001, de acordo com a Companhia de Entrepostos e Armazéns Gerais de São Paulo (CEAGESP), o volume comercializado de tomate para consumo foi de 152 mil t, com preço médio de US\$ 0,28/kg (FNP CONSULTORIA \& COMÉRCIO, 2001). Após a colheita, o tomate apresenta-se como um fruto altamente perecível. O fruto maduro possui vida média de prateleira de uma semana, com perdas variando entre $25 \mathrm{e}$ $50 \%$, enquanto o fruto parcialmente maduro apresenta vida útil de até duas semanas, com 20 a $40 \%$ de perdas pós-colheita (BARRET REINA, 1990). Mukai e Kimura (1986) também observaram em estudo que as perdas em tomate varia- ram de 0 a $50 \%$ dependendo do tipo de varejo e da época do ano. As perdas são maiores na época chuvosa. Por sua vez, Tsunechiro et al., (1994) relatam que no mercado varejista em São Paulo as perdas variaram de 14\% em 1976 para $11,8 \%$ em 1991. Lana et al. (1999) relatam que as perdas de tomates ocorridas em uma rede de supermercados variam de 5 a $25 \%$. A grande maioria deve-se a perdas por dano mecânico $(55,6 \%)$ e também por dano fisiológico $(4,6 \%)$.

As perdas pós-colheita em frutas e hortaliças são causadas principalmente por injúrias mecânicas, como, manuseio e transportes inadequados, armazenamento impróprio, e grande tempo de exposição no varejo (CEAGESP, 2002). Injúria mecânica pode ser causada por mais de um tipo de força: impacto, compressão e vibração (BRUSEWITZ et al., 1991;
VERGANO et al., 1991). Os impactos ocorrem na queda do fruto (GARCIA et al., 1988). A compressão ocorre no contato entre frutos, por exemplo, embalagens lotadas de frutos (BANKS et al., 1991) ou também durante a colheita, devido à pressão dos dedos. A vibração decorre de repetitivos impactos em baixa freqüência entre frutos (MANESS et al., 1992), ocorrendo geralmente durante o transporte, causando alta porcentagem de perdas (JONES et al., 1991). Hortaliças e frutas reagem diferentemente aos danos físicos (DELWICHE et al., 1989). Sommer et al., (1960) relatam que peras são muito mais sensíveis ao impacto do que às vibrações. Outros frutos como morangos e maçãs, são mais sensíveis à compressão (HOLT; SCHOORL, 1976; HOLT; SCHOORL, 1982).

Danos fisiológicos e patológicos se dão principalmente, na fase de produ-

\footnotetext{
${ }^{1}$ Parte do projeto de pesquisa UNIMAC (Unidade Mecânica de Auxílio à Colheita) financiado pela FAPESP.
} 
ção, transporte e armazenamento. As injúrias causadas por impactos, compressões, vibrações, cortes e rachaduras estão relacionadas com alterações fisiológicas, metabólicas, de aroma, sabor e qualidade em diferentes produtos hortícolas, tais como, maçãs, pepinos, batatas e tomates (MORETTI; SARGENT, 2000). A grande incidência de danos físicos em tomate acontece durante a colheita, aumentando durante o transporte e beneficiamento (FERREIRA et al., 2003).

Silva e Calbo (1991) avaliaram a colheita de tomate empregando diversos acessórios e concluíram que a sacola de colheita usada em outros países (Estados Unidos e Canadá) possibilitou maior rendimento e menores danos aos frutos, com menor impacto. No entanto, no Brasil, a colheita de tomates para consumo in natura é realizada utilizando-se cestas de bambu e transferindo os frutos para caixas plásticas. Alguns produtores, em busca de uma maior rapidez para o processo de colheita utilizam sacolas de colheita confeccionadas com lona plástica originalmente usada para laranja, podendo ser uma alternativa para colheita do tomate. Porém, o efeito desta modificação no processo na qualidade final dos frutos de tomate nas nossas condições de cultivo ainda é desconhecido.

O objetivo deste trabalho foi avaliar técnicas de colheita utilizando-se cesta de bambu e sacola de lona plástica, na qualidade de tomate cv. Débora.

\section{MATERIAL E MÉTODOS}

Foram utilizados frutos de tomate, cultivar Débora (Sakata seeds) colhidos em propriedade rural, situada no município de Estiva Gerbi (SP), coordenadas geográficas $46^{\circ} 53^{\prime} \mathrm{W}$ e $22^{\circ} 19^{\prime} \mathrm{S}$, com temperatura média anual de $19,6^{\circ} \mathrm{C}$ e precipitação anual de $1565 \mathrm{~mm}$.

O cultivo foi em Latossolo Vermelho Amarelo em sistema tradicional de plantio, conduzida em estaca de bambu, com o uso de irrigação por sulcos, utilizando-se espaçamento entre linhas de $1,00 \mathrm{~m}$ e entre plantas de $0,50 \mathrm{~m}$. Frutos foram colhidos primeiramente em cesta de bambu, com dimensões externas de $40 \mathrm{~cm}$ de comprimento, $25 \mathrm{~cm}$ de altura e $20 \mathrm{~cm}$ de largura e após seu enchimento foram transferidos para caixa plástica com dimensões de $55 \mathrm{~cm}$ de comprimento, $30 \mathrm{~cm}$ de altura e $35 \mathrm{~cm}$ de largura, posicionada no carreador principal de plantio. Em outra situação, a colheita foi realizada utilizando-se sacolas de lona plástica (utilizadas para colheita de laranja), com dimensões externas de $65 \mathrm{~cm}$ de comprimento e $55 \mathrm{~cm}$ de largura. Em seguida, os frutos foram transferidos para caixas plásticas, semelhante às do sistema de colheita em cestas de bambu. O tempo gasto para colheita foi mensurado nas duas situações utilizando-se cronômetros de precisão.

Em cada caixa nos dois sistemas mencionados foi retirada amostra de 40 frutos no estádio salada (CEAGESP, 2000). A testemunha correspondia a frutos (40) retirados diretamente da planta, sem passar pelo manuseio descrito anteriormente. Foram retiradas amostras de 3 caixas, sendo cada caixa uma repetição em um total de 120 frutos por tratamento. Estes frutos foram transportados cuidadosamente em embalagens de papelão, envoltos individualmente em espuma para o laboratório da UNICAMP e armazenados à temperatura ambiente $\left(23^{\circ} \mathrm{C}\right)$ por 21 dias.

Foram avaliados os danos físicos (\%) baseando-se nas Normas e Padrões de Classificação (CEAGESP, 2000) identificando os em duas categorias: antes e durante a colheita. As injúrias mecânicas anteriores à colheita foram caracterizadas como: derivadas de abrasão com as estacas de bambu e fios de amarrio, ataque de insetos e distúrbios fisiológicos e nutricionais; as injúrias mecânicas causadas na colheita foram identificadas como: derivadas de compressão do fruto contra a cesta de colheita e/ou caixa plástica, impacto do fruto quando em queda na caixa plástica e/ou cesta de colheita, marcas de unhas ou dedos e compressão do pedúnculo do fruto contra superfície externa de outro fruto. O diâmetro transversal do fruto foi mensurado utilizandose paquímetro digital. Para mensuração da área externa referente ao dano físico, estas foram delimitadas utilizando-se caneta marcador para retroprojetor de cor pre- ta. Após a delimitação das áreas referentes a danos físicos, as marcações foram transferidas para papel de seda e individualizadas por fruto. Para mensurar estas áreas utilizou um planímetro (KEUFFEL \& ESSER Co). As áreas mensuradas foram comparadas à superfície total do fruto, considerando este com uma esfera (MOHSENIN, 1986) e utilizando-se a seguinte fórmula: Área Externa do fruto $=4 \times 3,1415 \times \mathrm{R}^{2}$ ( $\mathrm{R}=$ raio) sendo o resultado expresso em porcentagem. Utilizou-se a mesma metodologia em duas categorias (antes e durante a colheita) em todos os tratamentos. A perda de massa (\%) foi avaliada a cada quatro dias utilizando a relação entre a diferença da massa inicial e final.

Após 21 dias de armazenamento, os frutos foram avaliados quanto à aparência encontrada, baseando-se em uma escala de $0-5$, considerando (0) fruto apropriado para consumo; (1) descarte por dano físico externo superficial; (2) descarte por dano físico externo grave; (3) descarte por dano físico e podridão associada (4) descarte por podridão; (5) descarte por perda de água (seco) ou por queima do sol. Considerou-se frutos apropriados para consumo aqueles que não apresentavam nenhum dano externo e/ou podridões ou danos físicos externos leves inferiores a $10 \%$. Tomates foram descartados devido a danos físicos externos leves e graves, quando a incidência foi superior a $10 \%$, sendo que a presença de injúrias graves era fator de exclusão principal. Considerou-se podridões causadas por fungos e/ou bactérias em qualquer nível. Tomates secos e/ou queimados de sol, eram aqueles que demonstravam queimaduras e/ou perda de água excessiva.

O delineamento utilizado foi inteiramente casualizado em esquema fatorial (sistemas de colheitas x dias após a colheita). O teste estatístico realizado para avaliação da qualidade após armazenamento foi o da diferença de proporções (BUSSAB; MORETTIN, 2002), em nível de 5\% de probabilidade. Os resultados médios obtidos para danos físicos foram comparadas pelo teste Tukey ao nível de 5\% de probabilidade e para perda de massa foram realizadas análises de regressão no desdobramento da interação dentro de cada sistema de colheita. 
Tabela 1. Avaliação para danos físicos (\%) em frutos de tomate cultivar 'Débora', em diferentes técnicas de colheita (cesta e sacola plástica) comparadas a testemunha. Campinas, UNICAMP, 2003.

\begin{tabular}{lc}
\hline Técnica de colheita & Dano físico (\%) \\
\hline Testemunha & $1,27 \mathrm{~b}$ \\
Cesta & $3,55^{\star} \mathrm{a}$ \\
Sacola & $3,99 \mathrm{a}$ \\
\hline
\end{tabular}

* Médias seguidas pela mesma letra não diferem significativamente pelo teste de Tukey a $5 \%$ de probabilidade

Tabela 2. Avaliação de descarte de frutos após 21 dias de armazenamento, baseado em escala 0-5, considerando (0) fruto apropriado para consumo; (1) descarte por dano físico externo superficial; (2) descarte por dano físico externo grave (3) descarte por dano físico e podridão associadas (4) descarte por podridão; (5) descarte por perda de água (seco) ou por queima do sol. Campinas, UNICAMP, 2003.

\begin{tabular}{|c|c|c|c|c|c|c|c|}
\hline \multirow{2}{*}{$\begin{array}{l}\text { Técnica de } \\
\text { colheita }\end{array}$} & \multicolumn{6}{|c|}{ Escala de notas (\%) } & \multirow{2}{*}{$\begin{array}{c}\text { Total } \\
\text { descarte }(\%)\end{array}$} \\
\hline & 0 & 1 & 2 & 3 & 4 & 5 & \\
\hline Testemunha & 62,50 & 0 & 5,00 & 7,50 & 20,00 & 5,00 & 37,50 \\
\hline Cesta & 28,34 & 5 & 42,50 & 3,33 & 15,00 & 5,83 & 71,66 \\
\hline Sacola & 17,50 & 5 & 40,00 & 8,33 & 26,67 & 2,50 & 82,50 \\
\hline Test. x Cest. & $s^{*}$ & $\mathrm{~s}$ & s. & n.s. & n.s. & n.s. & $\mathrm{s}$ \\
\hline Test. x Sac. & s & $\mathrm{s}$ & $\mathrm{s}$ & n.s. & n.s & n.s. & s \\
\hline Cesta x Sac. & $\mathrm{s}$ & n.s & n.s. & n.s. & $\mathrm{s}$ & n.s. & n.s \\
\hline
\end{tabular}

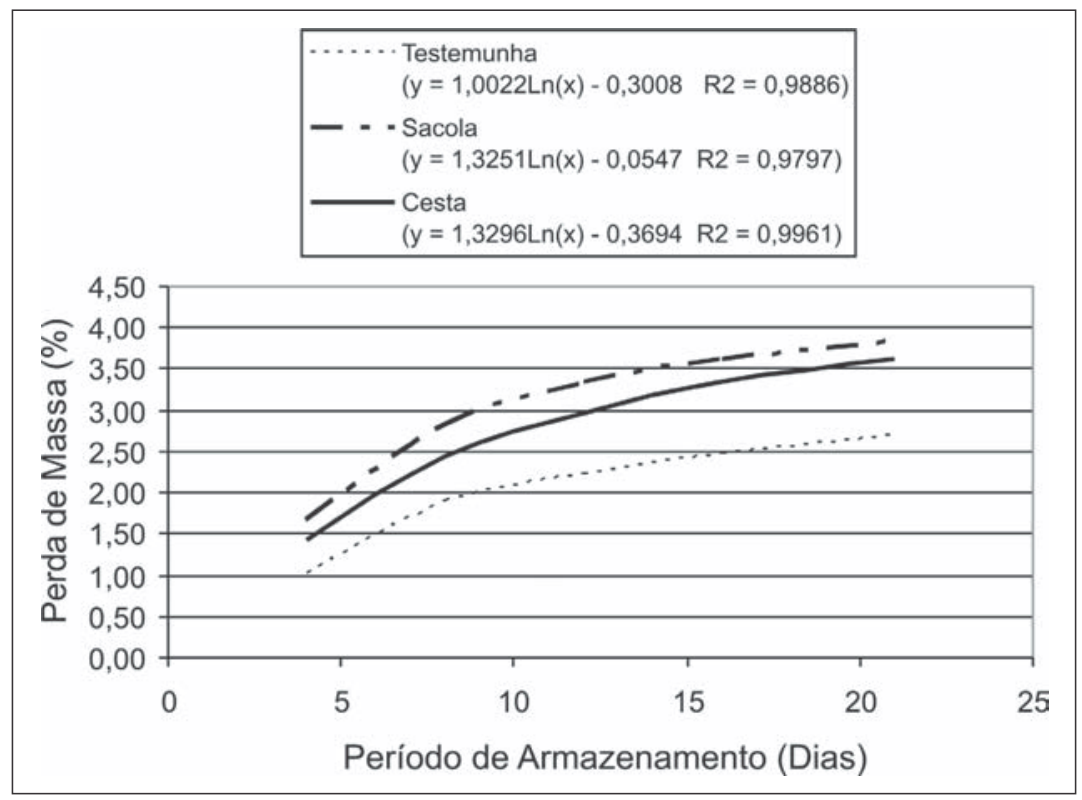

Figura 1. Modelos ajustados para a perda de massa, em porcentagem, (y) em função dos períodos de armazenamento, em dias, (x) dentro de cada sistema de colheita. Campinas, UNICAMP, 2003.

\section{RESULTADOS E DISCUSSÃO}

O tempo necessário para realizar a colheita no mesmo número de plantas utilizando-se cesta de bambu foi de
20'13", superior em $20 \%$, em relação a sacola plástica $\left(15^{\prime} 51^{\prime \prime}\right)$. Porém, este menor tempo de colheita não interferiu positivamente na qualidade final do fruto. Silva e Calbo (1991) em avaliação de diferentes métodos de colheita para tomate de mesa, também encontraram maior rendimento com o uso da sacola de colheita, todavia com menores danos físicos aos frutos.

Observou-se maior incidência de danos físicos, ainda que não significativo, em tomates colhidos com sacola de lona plástica $(3,99 \%)$, quando comparados àqueles colhidos com cestas de bambu $(3,55 \%)$ (Tabela 1). Os tomates colhidos sem esse manuseio (testemunha) apresentaram 1,27\% de danos físicos. Esses frutos apresentavam danos físicos relacionados ao ataque de insetos, e estacas de bambu, demonstrando que as perdas já iniciam no campo e aumentam na etapa de colheita, como também foi observado por Sanches (2002) em bananas. Hung e Prussia (1989) também relatam que a incidência de danos físicos está relacionada ao tipo e intensidade do manuseio utilizado.

De acordo com Schoorl e Holt, (1982), os danos físicos causados por impacto e compressão são os que mais influenciam na qualidade dos frutos de tomate. Na colheita de tomates, o ponto crítico para incidência em danos físicos ocorreu principalmente quando do impacto do fruto em queda na caixa plástica e/ou cesta de colheita e a compressão do fruto contra a superfície externa da cesta ou sacola plástica. Altura e velocidade de queda, enchimento das caixas, cestas e sacolas plásticas, são fatores que ocasionaram danos aos frutos. Treinamento de funcionários e utilização de equipamento adequado podem modificar a situação encontrada.

Observa-se perda de massa (Figura 1) crescente durante o armazenamento, porém significativamente maior em tomates colhidos com sacola e cesta de bambu em relação à testemunha.

A qualidade dos frutos no final de 21 dias de armazenamento foi comprometida principalmente em frutos colhidos com sacola $(82,5 \%$ de descarte) quando comparada com colheita utilizando cesta de bambu $(71,66 \%)$, provavelmente devido a maiores danos físicos associados a podridões. A testemunha apresentou $37,5 \%$ de frutos descartados após armazenamento. Os danos físicos graves provenientes da etapa de colheita foram as principais cau- 
sas de descarte, principalmente para frutos colhidos com cesta $(42,5 \%)$ e sacola $(40 \%)$ (Tabela 2).

Silva e Calbo (1991) relataram que frutos colhidos com sacola plástica apresentaram menores danos quando comparados a outros métodos, como por exemplo, a colheita com a utilização de cestas. Uma razão para isto deve-se ao uso de sacolas de colheita com estrutura de sustentação, o que proporciona menor força de compressão, pois maiores forças de compressão causam maiores perdas (BANKS et al., 1991). A colheita utilizando-se sacolas plásticas foi realizada em menor tempo, todavia com maiores perdas e redução na qualidade final do produto. A colheita utilizandose sacolas plásticas sem sustentação não se mostrou adequada, influenciando negativamente na qualidade final dos frutos após o armazenamento. Essas maiores perdas podem estar relacionadas a uma maior força de compressão entre os frutos. Observa-se que métodos alternativos de colheita devem ser estudados visto que neste experimento causaram grande redução na qualidade final do produto, além disso, deve haver maior preocupação com os frutos antes da colheita, o que também é responsável por elevadas perdas.

\section{AGRADECIMENTOS}

Os autores agradecem à FAPESP e ao proprietário e funcionários da empresa MALLMANN, Mogi-Guaçu (SP).

\section{LITERATURA CITADA}

BANKS, N.K.; BORTON, C.A.; JOSEPH, M. Compression bruising test for bananas. Journal of Science Food Agriculture, v.56, p.223-226, 1991.
BARReTt ReINA, L.C. Conservação Pós-colheita de Tomate (Lycopersicon esculentum. Mill) da Cultivar Gigante Kada Submetido a Choque a Frio e Armazenamento com Filme de PVC. 1990. 114 f. Dissertação (mestrado em Ciência dos Alimentos) - Faculdade de Ciência dos Alimentos, Escola Superior de Agricultura de Lavras, Lavras. BUSSAB, W.O.; MORETTIN, P. Estatística básica. 5a edição, São Paulo. Saraiva, 2002. 321 p. BRUSEWITZ, G.H.; McCOLLUM; T.G.; ZHANG, X. Impact bruise resistance of peaches. Transactions of the ASAE, v.34, n.3, p.962-965, 1991.

CEAGESP. Classificação de Tomate. São PauloSP, Programa Horti\&Fruti, 2000. 3p.

CEAGESP. Diga não ao desperdício. Disponível Em: 〈http://www.ceagesp.com.br>. Acesso em: 15 jul. 2002.

DELWICHE, M.J.; TANG, S; MELHSCHAU, J.J. An impact force response fruit firmness sorter. Transactions of the ASAE, v.32, n.1, p.321-326, 1989.

FERREIRA, M.D., FRANCO, T.O.A., KASPER, R.F., NOGUEIRA, M.F.M., CHIUMARELLI, M. Avaliação da qualidade do tomate de mesa, cv. 'Romana' em diferentes etapas na pós-colheita. Resumo Expandido. CD Anais. Horticultura Brasileira, v.21, n.2. 4 p., Julho 2003.

FNP Consultoria \& Comércio. Anuário da Agricultura Brasileira - AGRIANUAL 2002. São Paulo:FNP, 2001, $536 \mathrm{p}$.

GARCIA, C., RUIZ, M.; CHEN, P. Impact parameters related to bruising in selected fruits. American Society of Agricultural Engineers Meeting, $\mathrm{n}_{\mathrm{o}}$. 88-607, 16 p. 1988.

HOLT, J.E; SCHOORL, D. Bruising and energy dissipation in apples. Journal of Texture Studies, v.7, p.421-432, 1976.

HOLT, J.E; SCHOORL, D. Strawberry bruising and energy dissipation. Journal of Texture Studies, v.13, p.349-357, 1982

HUNG, Y.C.; PRUSSIA, S.E. Effect of maturity and storage time on the bruise susceptibility of peaches (cv. Red Globe). Transactions of the ASAE, v.32, n.4, p.377-1382, 1989

JONES, C.S.; HOLT, J.E.; SCHOORL, D. Model to predict damage to horticultural produce during transport. Agriculture Engineering Research, v.50, p.259-272, 1991.
LANA, M.M.; MOITA, A.W.; NASCIMENTO, E.F.; SOUZA, G.S.; MELO, M.F. Quantificação e caracterização das perdas pós-colheita de tomate no varejo. Horticultura Brasileira, Brasília, v.17, n.3, p.295, 1999.

MANESS, N.O.; BRUSEWITZ, G.H.; McCOLLUM, T.G. Impact bruise resistance comparison among peach cultivars. HortScience, v.27, n.9, p.1008-1011, 1992.

MOHSENIN, N.N. Physical properties of plant and animal materials. Vol. I. New York:Gordon Beach Science Publishers, 1986, 841 p.

MORETTI, C.L.; SARGENT, S.A. Alteração de sabor e aroma em tomates causada por impacto. Scientia Agrícola, Piracicaba, v.57, n.3, p.385-388, 2000.

MUKAI, M.K.; KIMURA, S. Investigação das práticas pós-colheita e desenvolvimento de um método para análise de perdas de produtos hortícolas. Viçosa: CENTREINAR, 1986.

SANCHES, J. Qualidade pós-colheita de bananas (Musa cavendishii) 'nanicão', através da classificação de defeitos físicos, embalagens e tecnologia do frio. 2002. 82 f. Dissertação (Mestrado em Tecnologia Pós-Colheita) - Faculdade de Engenharia Agrícola, Universidade Estadual de Campinas.

SCHOORL, D.; HOLT, J.E. Impact bruising in 3 apple pack arrangements. Journal Agricultural Engineering Research, v.27, p.507-512, 1982.

SILVA, J.L.O.; CALBO, A.G. Comparação entre métodos de colheita de tomate. Horticultura Brasileira, Brasília, v.9, p.58, 1991

SOMMER, N.F.; MITCHELL, F.G.; GUILLOU, R.; LUVISI, D.A. Fresh fruit temperature and transit injury. Proceedings American Society for Horticultural Science, v.76, p.156-162, 1960.

TSUNECHIRO, A.; UENO, L.H.; PONTARELLI, C.T.G. Avaliação econômica das perdas de hortaliças e frutas no mercado varejista da cidade de São Paulo, 1991/1992, Agricultura em São Paulo, SP, v.41, n. 2, p. 1-15, 1994.

VERGANO, P.J.; TESTIN, R.F.; NEWAL JR., W.C. Peach bruising: susceptibility to impact vibration, and compression abuse. American Society of Agricultural Engineers, v.34, n.5, p.2110-2116, 1991. 